\title{
A study on compatibility and interoperability among multi- GNSS
}

\begin{abstract}
Background: During the last decade replenishment and modernization of 2 global satellite navigation systems are witnessed. Development and deployment of another 2 global navigation systems are also observed. 2 regional satellite navigation systems are emerging fast along with them. Now there are more than 120 satellites available and dedicated for navigation purpose. This situation is termed as 'Multi- GNSS'. 'Multi- GNSS' can provide uninterrupted position solution under constraint conditions to the users. But for using 'Multi- GNSS' more effectively and efficiently users should properly investigate the issues of interoperability and compatibility among the systems. This paper will address this issue of interoperability and compatibility between different GNSS systems for having the full benefits of using all the systems together.
\end{abstract}

Volume 5 Issue I - 202|

\author{
Shreya Sarkar ${ }^{\prime}$ \\ 'Burdwan Town School, India
}

Correspondence: Shreya Sarkar, Burdwan Town School, Court Compound, Burdwan-7/3।0I, District-Purba Bardhaman, West Bengal, India, Tel +91 9475484779,

Email shreya.sarkar@community.isunet.edu

Received: April 20, 2021 | Published: May 03, 2021

Keywords: multi-GNSS, interoperability, compatibility

\section{Introduction}

Global Positioning System (GPS) and GLObal'naya NAvigatsionnaya Sputnikovaya Sistema (GLONASS) developed by United States Department of Defence (USDoD) and by the space forces of Soviet Russia, respectively. BeiDou and Galileo were developed by China and European Union (EU), respectively. The main purpose of development of Galileo is for civilian use. 2 regional satellite systems Navigation with Indian Constellation (NavIC) and Quasi Zenith Satellite System (QZSS) were developed and deployed by India and Japan, respectively. Currently, 3 global satellite-based navigation systems are declared as fully deployed and operational (GPS, GLONASS and BeiDou) and Galileo is under development stage. ${ }^{1-4}$ But these systems are developed individually for serving the individual need of their country. GLONASS is emerged as an independent system and replenished after a degradation about a decade, whereas, GPS is rather a fully developed consistent service provider since deployment. BeiDou is independently developed. Galileo is developing with a declaration as being compatible to GPS. None of the systems uses same coordinate frame of reference and time frame of reference. The signal structure for GLONASS is also unlike the other systems. These lead to the problem of compatibility and interoperability among the systems.

Interoperability is defined as the ability of global, regional and their augmentation systems when used together for providing better solution accuracy rather than relying on any of the stand- alone system. Interoperability is mainly divided in two types. 1. System level and 2. Signal level. It is defined as the capability of different GNSS system to provide same level of accuracy while used as stand- alone. e.g., Galileo is claimed to be interoperable with GPS in system level. ${ }^{5}$ Signal level interoperability is defined as the similarity of signals among different GNSS. It is divided in four categories depending on the following factors. 1. Reference frame, 2. Time reference, 3. Carrier frequency and 4. Signal in Space. ${ }^{6}$ The standard civil coordinate reference frame is International Terrestrial Reference Frame (ITRF). But there are different reference coordinate frames used by different GNSS systems. GPS system uses World Geodetic System (WGS84), GLONASS uses Parametry Zemli 1990 (PZ90), Galileo uses Galileo Terrestrial reference Frame (GTRF), BeiDou uses China Geodetic Coordinate System 2000 (CGCS2000), QZSS adopts Japan satellite navigation Geodetic System (JGS), NavIC uses WGS84 and all currently available Satellite Based Augmentation Systems (SBAS) adopt WGS84 as they have emerged to augment GPS. Thus, NavIC is interoperable with GPS with respect to reference frame. GTRF claimed to differ by $3 \mathrm{~cm}$ from WGS $84 .{ }^{6}$ The standard reference time is Universal Time Coordinated (UTC). GPS System Time (GPST) and GLONASS System Time (GLONASSST) use U.S. and Russian versions of UTC respectively. BeiDou Time (BDT) is offset by 100ns from UTC. QZSS Time (QZSST) follows UTC. Galileo uses Galileo System Time (GST) as reference time. There is nanosecond level offset between GPST and GST and this offset time is provided to the receiver beforehand, although some of the receivers consider this time as another unknown and solve it under navigation solution. Thus, Galileo is interoperable with GPS in terms of Time reference also. ${ }^{6}$ GPS and Galileo are interoperable with respect to carrier frequency as both use L1 and L5/ E5a under Code Division Multiple Access (CDMA) technique. Though the other frequency band L2 and E5b are not interoperable but they are compatible as they are not affected by interference. ${ }^{6}$ Signal structure, modulation technique requires software modification in receiver and not affect the interoperability among the systems. e.g., military GPS- M code is interoperable with Galileo civil signal on L1 band. QZSS is claimed to be interoperable with both GPS and Galileo in L1 and L5/E5a band. ${ }^{6}$

Compatibility is the ability of using the global, regional or their augmentation systems individually or together without adversely affecting the signals due to interference. A framework on GNSS compatibility is defined by the International Telecommunication Union (ITU). Several co-operations in national and international levels are initiated to properly address the issue of interoperability and compatibility. These agreements among the service providers are listed below:

1998: US-Japan agreement to make Multi- functional Satellite Augmentation System (MSAS) fully compatible and interoperable to GPS.

2003: EU-China agreement to organise technical meeting regularly to assure BeiDou compatible to Galileo. ${ }^{6}$ 
2004: Four working groups are established for GPS- GLONASS agreement. One is for addressing the issue of radio frequency compatibility and another one is working on technical compatibility between the systems. ${ }^{6}$

2004: EU-US agreement to make GPS and Galileo compatible with each other. As a consequence, all GNSS receivers made operable with Galileo from 2014 across the globe. ${ }^{6}$

2007: US-Australia co-operation is developed to guarantee the interoperability between GPS and Australia's Ground Based Augmentation System (GBAS).

2007: US-India's co-operation is initiated to make India's augmentation system GPS-aided GEO Augmented Navigation (GAGAN) interoperable with GPS. ${ }^{6}$

2017: A GNSS working group is also dedicated since 2017 to make QZSS compatible and interoperable with new generation GPS Block-III satellites.

Efforts are observed from researchers to develop a unique and suitable transformation tool to make the system interoperable with each other. ${ }^{8-10}$ Initial efforts were observed from the researchers to develop an interoperability between GPS and GLONASS as those systems were fully deployed and available initially. But a globally acceptable unique conversion tool had never been developed for these two systems. ${ }^{11,12}$ After the development of Galileo interests and emphasis were given more on using these two systems together as Galileo is claimed to be interoperable with GPS. ${ }^{13}$ But users and researchers never observed and established any interoperability technique between the two systems. Two regional systems NavIC and QZSS are claimed to be interoperable with GPS. But only GPS and NavIC are using a unique coordinate reference frame WGS84 so far. ${ }^{14}$ BeiDou uses its own reference coordinate and time coordinate unlike others..$^{15}$ The issue of compatibility among the systems are maintained by the frequency band allocation as provided and guided by International Telecommunication Union (ITU). ${ }^{16}$ But signal interference is also an issue responsible for degradation of position solution.

A comparison among the different available Global and Regional satellite systems are summarised in Table 1 and Table 2, respectively.

From Tables $1 \& 2$ it can be seen different systems are using different time reference, coordinate reference frames, frequency bands, multiplexing techniques. Therefore, this is important to verify the compatibility and interoperability levels among the systems before using them together with confidence.

This paper tries to analyze some obtained results from different capability receivers to observe if the systems are interoperable and compatible or not as claimed. Furthermore, this paper tries to identify whether using Multi- GNSS can always assure us with betterment in position solution.

Table I A comparative study among different global satellite navigation systems

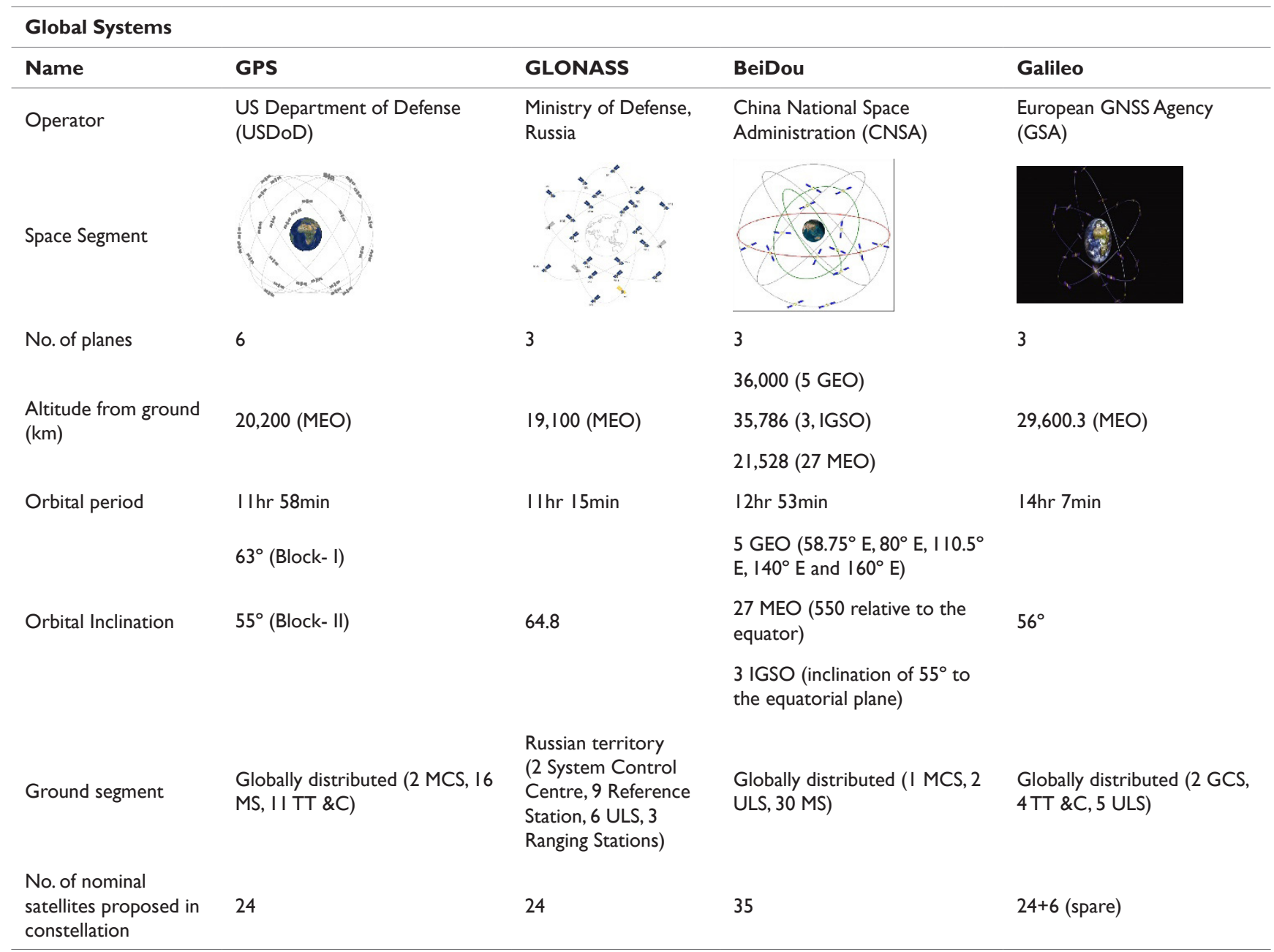


Table Continued..

\begin{tabular}{|c|c|c|c|c|}
\hline \multicolumn{5}{|l|}{ Global Systems } \\
\hline Name & GPS & GLONASS & BeiDou & Galileo \\
\hline \multicolumn{5}{|l|}{$\begin{array}{l}\text { No. of satellites } \\
\text { operational (in } \\
\text { constellation) }\end{array}$} \\
\hline Multiplex technique & CDMA & $\begin{array}{l}\text { FDMA, } \\
\text { CDMA }\end{array}$ & CDMA & CDMA \\
\hline \multirow{4}{*}{$\begin{array}{l}\text { Frequency bands } \\
(\mathrm{MHz})\end{array}$} & LI (I575), L2 (I 227), & LI (1602.0- I6I5.5), & $\mathrm{BIC/BII/BIA} \mathrm{(I575.42),}$ & EI (I559-I59I), \\
\hline & L5 (I I76), & L2 (I246.0- I256.5) & B2a/ B2b (I I9I.795), & E5 (I |64-12|4), \\
\hline & \multirow[t]{2}{*}{ LIC (I575) } & & B3I/ B3Q/ B3A (I268.52), & \multirow[t]{2}{*}{$E 6(1260-1300)$} \\
\hline & & & Bs (2492.028) & \\
\hline Reference Frame & WGS84 & PZ90 & CGCS2000 & GTRF \\
\hline Reference Time & GPST & GLONASSST & BDT & GST \\
\hline
\end{tabular}

Table 2 A comparative study among different regional satellite navigation systems

\begin{tabular}{|c|c|c|}
\hline \multicolumn{3}{|l|}{ Regional Systems } \\
\hline Name & QZSS & NaviC \\
\hline Operator & Japan Aerospace Exploration Agency (JAXA) & $\begin{array}{l}\text { Indian Space Research } \\
\text { Organization (ISRO) }\end{array}$ \\
\hline \multicolumn{3}{|l|}{ Space Segment } \\
\hline No. of planes & N/A & $\mathrm{N} / \mathrm{A}$ \\
\hline Altitude from ground $(\mathrm{km})$ & perigee 32,000 , apogee 40,000 ( $3 \mathrm{HEO}$ ) and 36,000 (I GEO) & $36,000(\mathrm{GEO})$ \\
\hline Orbital period & $23 \mathrm{hr} 56 \mathrm{~min}$ & $23 \mathrm{hr} 55 \mathrm{~min} 59 \mathrm{sec}$ \\
\hline & & $3 \mathrm{GEO}\left(32.5^{\circ} \mathrm{E}, 83^{\circ} \mathrm{E}, 129.5^{\circ}\right.$ \\
\hline Orbital Inclination & $39^{\circ} \sim 47^{\circ}$ & $\begin{array}{l}\text { E) } 4 \text { IGSO, } 2 \text { of them cross } \\
\text { equator at } 55^{\circ} \mathrm{E} \text { and other } \\
\text { two at } 111.75^{\circ} \mathrm{E}\end{array}$ \\
\hline Ground segment & Distributed over Japan (2 MCS, 7 CS, 30 MS) & Data Not available \\
\hline $\begin{array}{l}\text { No. of nominal satellites proposed in } \\
\text { constellation }\end{array}$ & 7 & II \\
\hline No. of satellites operational (in constellation) & $4(4)$ & $7(7)$ \\
\hline \multirow[t]{3}{*}{ Multiplex technique } & CDMA & CDMA \\
\hline & LI- C/A (I575.42), & L5 ( 1 I 76.45), \\
\hline & LIC (I575.42), & S (2492.08) \\
\hline \multicolumn{3}{|l|}{ Frequency bands (MHz) } \\
\hline & L5 ( 1176.45$)$ & \\
\hline Reference Frame & JGS & WGS84 \\
\hline Reference Time & QZSST & IRNWT \\
\hline
\end{tabular}




\section{Methods}

Two different types of experiments have been designed and performed using two different capability GNSS receivers: 1. Geodetic receiver and 2. Cost-effective receiver. Data are taken from one fixed location whose reference position is already fixed and known situated in Burdwan, West Bengal, India. The first experiment is performed using the geodetic GNSS receiver and data are collected in different modes, both individual and hybrid modes with a 1 hour $(3600 \mathrm{sec})$ of succession in each mode by switching the receiver among different modes. In the second experiment two different capability receivers are operated in same modes simultaneously to observe the difference in solution accuracy due to the internal performance capability of the receiver.

\section{Results}

It is observed and reported that users need to depend on MultiGNSS for getting an uninterrupted position solution under constraint conditions like urban canyons, open cut mines, deep foliage etc. ${ }^{15}$ But more system does not always guarantee about the betterment of solution accuracy. Claims are made on status of interoperability and compatibility among the systems as found from the documentations and resolutions taken during different annual meetings conducted by the International Committee on Global Navigation Satellite System (ICG). But use of best possible Multi- GNSS scenario had never been verified and established. As mentioned in section 2, two different capability GNSS receivers are connected to the same reference position antenna using one splitter. The other end of the receivers is connected to two different laptops as shown in Figure 1.

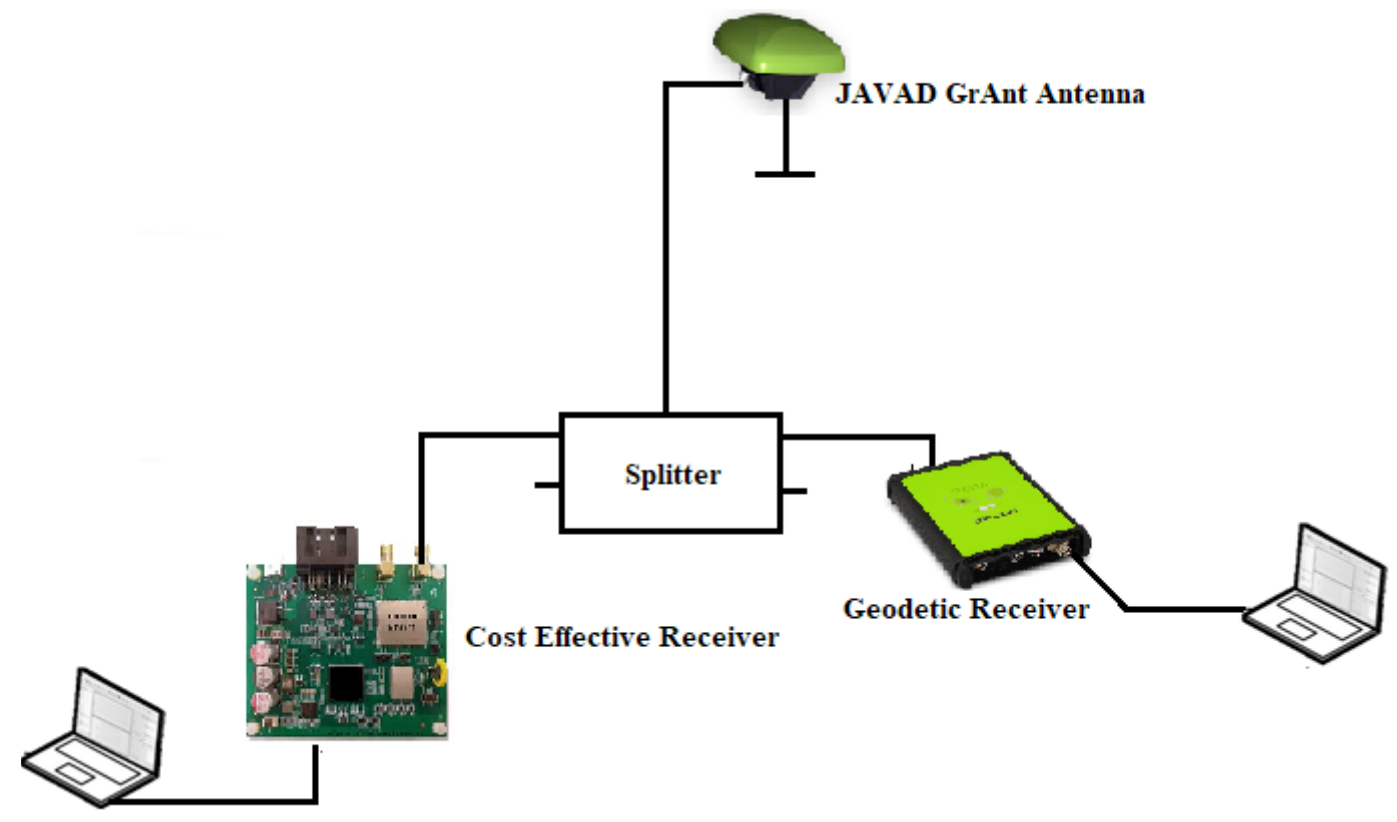

Figure I Experimental set-up.

Data are taken at $1 \mathrm{~Hz}$ rate for 1 hour in each mode (GPS, NavIC, GLONASS, GPS+GLONASS, Galileo and GPS+GLONASS+Galileo respectively) using the Geodetic receiver and the 2-dimensional (2-D) error in position solution or the horizontal error is calculated in each mode using Equation 1. ${ }^{18}$ The same experiment is repeated for GPS, Galileo, and GPS+Galileo modes on a different date using the same Geodetic receiver. The deviation in solution accuracy is calculated from the reference predefined antenna position $\left(23^{\circ} 15.16^{\prime} \mathrm{N}, 87^{\circ}\right.$ $50.49^{\prime}$ E). Obtained results are shown and discussed in section 4.

$$
\text { Error }_{2 d}=\sqrt{\left(1852 \cdot \Delta L_{t} \cdot \cos \left(L_{a 0}\right)\right)^{2}+\left(1852 . \Delta L_{a}\right)^{2}}
$$

Where, 1minute of arc of curvature of Earth is equivalent to $1852 \mathrm{~m}$.
In a second type of experimental set up two different capability receivers are operated in different modes (GPS, NavIC, GLONASS, GPS+GLONASS, Galileo and GPS+GLONASS+Galileo respectively) simultaneously as mentioned in section 2. Data are collected@1 $1 \mathrm{~Hz}$ data rate continuously for $1 \mathrm{hr}$ in each mode. The transition among the modes are done by switching the receivers to the next modes simultaneously. The observed average of 2-dimensional (2-D) errors are shown in Table 3.

A second set of similar experiment is repeated on a different date by switching the two receivers simultaneously in GPS, Galileo, and GPS+Galileo modes as GPS and Galileo are claimed to be interoperable and compatible with each other. The observed results are shown in Table 4.

Table 3 Average 2-D position errors in different modes in meters (28/I2/2019)

\begin{tabular}{lllllll}
\hline Receiver type & GPS & GLONASS & Galileo & NavIC & GPS+GLONASS & GPS+GLONASS+Galileo \\
\hline Geodetic & 0.76 & 2.67 & 1.54 & 4.63 & 1.64 & 0.95 \\
Cost- effective & 0.8 & 3.3 & 2.09 & 2.81 & 1.94 & 1.39 \\
\hline
\end{tabular}


Table 4Average 2-D position errors in different modes in meters $(01 / 01 / 2020)$

\begin{tabular}{llll}
\hline Receiver type & GPS & Galileo & GPS+Galileo \\
\hline Geodetic & $2.4 \mathrm{I}$ & $\mathrm{I} .8$ & $\mathrm{I} .7$ \\
Cost- effective & 0.79 & $\mathrm{I} .93$ & $\mathrm{I} .09$ \\
\hline
\end{tabular}

From the critical observation of Tables 3 and Table 4 it can be inferred that under open sky with all possible available constellations position solution accuracy is better with a repetitive nature. Although GPS stand- alone accuracy is better for low- cost applications but the situation can never be guaranteed.
Standard deviation/Jitter in Error values are shown in Table 5 and Table 6 respectively.

From Table 5 and Table 6 it can be inferred that the standard deviation of error value is worse for NavIC for the first set of experiment, whereas, it is worse for Galileo stand- alone mode of operation for the second set of experiment. But the values of standard deviation of errors are moderate for the Multi- GNSS scenario for both cases. But the level of interoperability and compatibility among the systems are varying widely with electronic and temporal variation. The results will be more minutely discussed under section 4 .

Table 5 Jitter in 2-D position errors in different modes in meters $(28 / 12 / 2019)$

\begin{tabular}{lllllll}
\hline Receiver type & GPS & GLONASS & Galileo & NavIC & GPS+GLONASS & GPS+GLONASS+Galileo \\
\hline Geodetic & 0.28 & 0.69 & 0.62 & 1.71 & 0.18 & 0.31 \\
Cost- effective & 0.34 & 1.36 & 0.84 & 2.3 & 0.5 & 0.41 \\
\hline
\end{tabular}

Table 6 Jitter in 2-D position errors in different modes in meters (0 I/0 I/2020)

\begin{tabular}{llll}
\hline Receiver type & GPS & Galileo & GPS+Galileo \\
\hline Geodetic & 0.37 & 0.49 & 0.28 \\
Cost- effective & 0.41 & 0.92 & 0.48 \\
\hline
\end{tabular}

The graphical representations of Experiment 1 as discussed in Section 5 are shown in Figure 2 and Figure 3, respectively.

GPS only position solution accuracy is better and within $1.5 \mathrm{~m}$ range but is unstable at the same time. 2-D position solution accuracy is within $1.5 \mathrm{~m}$ and $2 \mathrm{~m}$ in GPS+GLONASS+Galileo and GPS+GLONASS modes respectively with a stability in solution. But as the transition among the systems is not continuous so it can be interpreted that there is lack of interoperability and compatibility among the systems.

When the same experimental set- up is repeated on a different day GPS only position solution accuracy becomes even worse than Galileo unlike the previous situation. But again, position solution accuracy gets better in GPS+Galileo mode and it is under $2 \mathrm{~m}$. The transition between the modes is not continuous which leads to improper interoperability and compatibility among the systems.

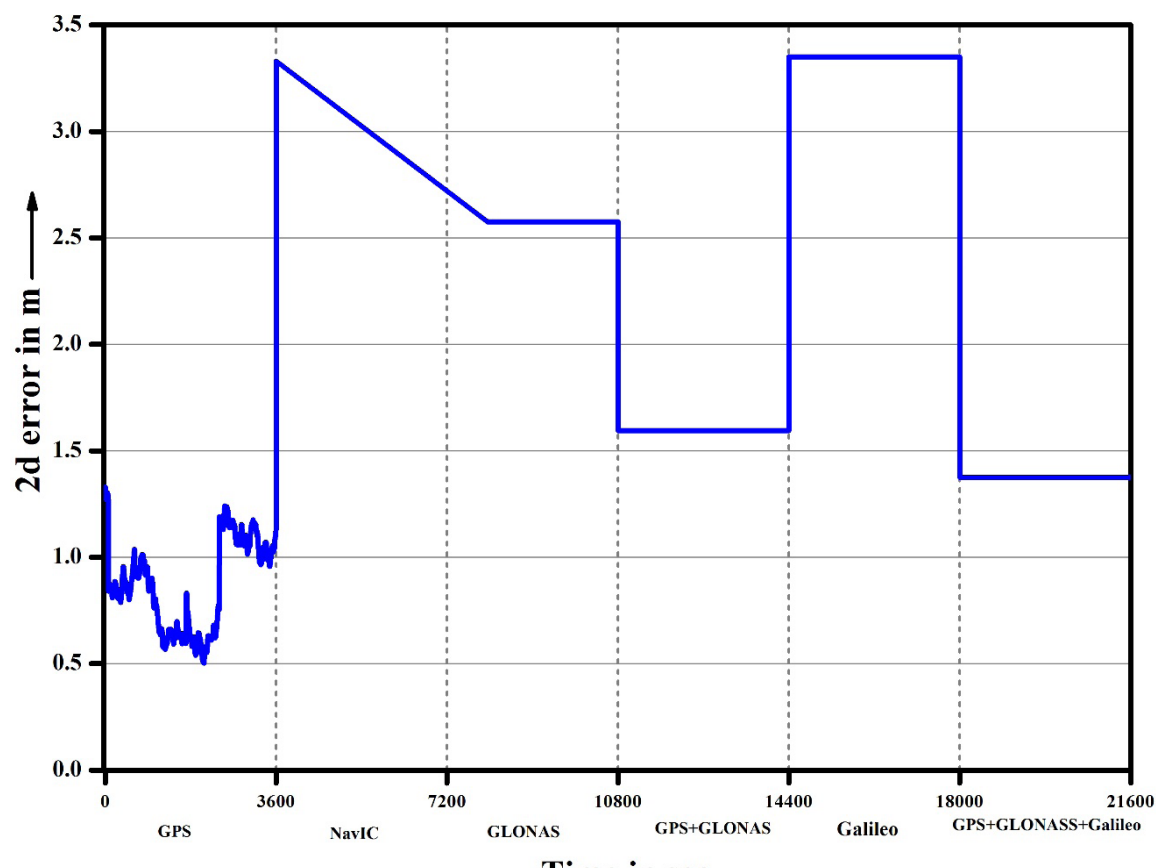

Time in sec $\longrightarrow$

Figure 2 2-D error in different modes using geodetic receiver (28/I2/20I9). 


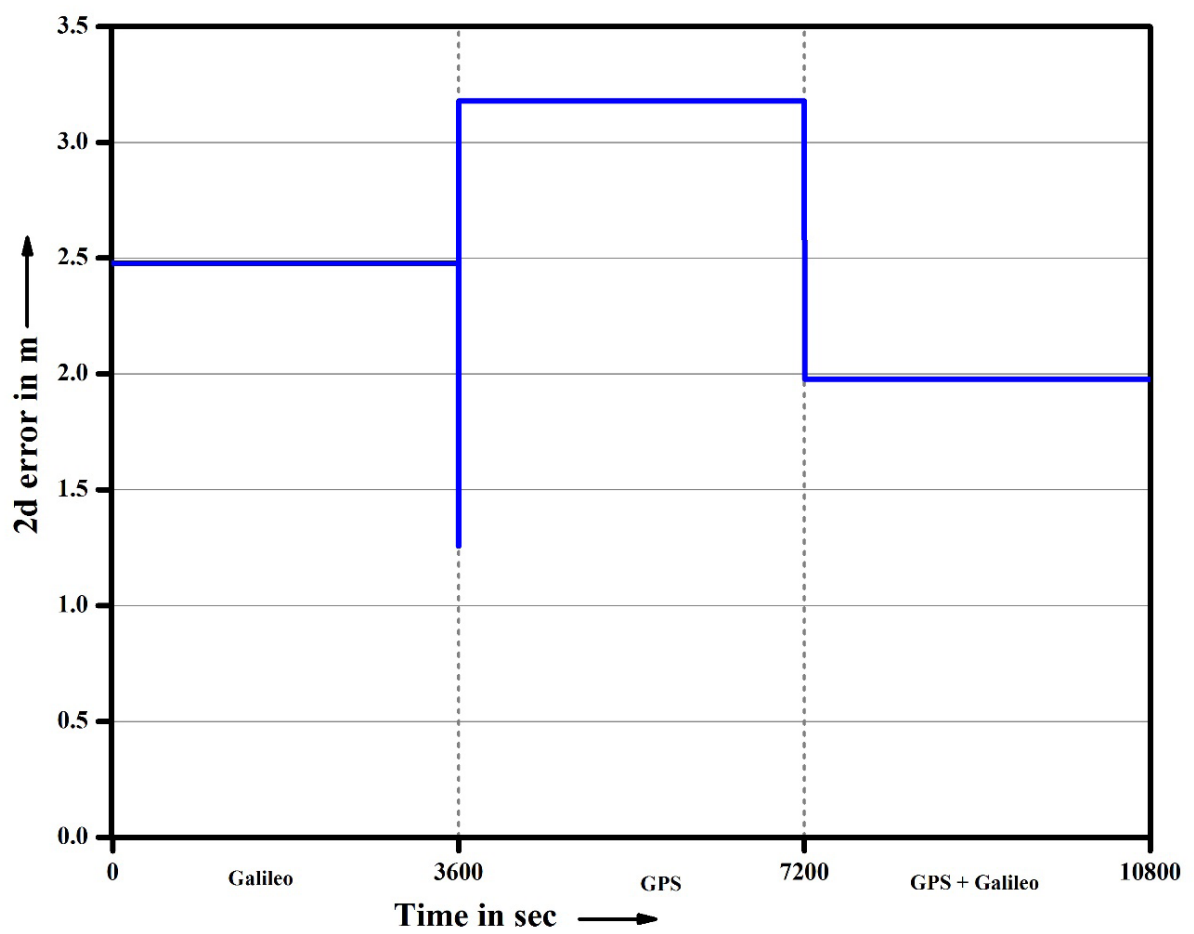

Figure 3 2-D error in different modes using geodetic receiver (0I/0I/2020).

\section{Discussion}

Interoperability and compatibility are the key issues to be taken care of for having the myriads of benefits of using Multi- GNSS. This issue is particularly important for system developers, service providers, researchers, and users. The utmost important daily life applications are highly dependent on seamless, robust, and uninterrupted position solution accuracy. GPS is still proving its superiority over other systems for low cost applications. GPS+GLONASS was better solution provider that can be used with much confidence but with the development and inclusion of Galileo the situation is changing towards a much betterment. During the observation of the obtained results a dependence on electronic and temporal variation is observed. A more proper interoperability and compatibility tool between the systems need to be developed properly.

\section{Conclusion}

The interoperability among different GNSS systems are observed and maintained in national and international levels under the supervision of ICG. Compatibility among the systems are maintained by the regulations and spectrum allocation defined by ITU. But from the observed results it can be inferred that the lack of continuous transition among the systems could be a result of deficiency in interoperability and compatibility among the systems. Newly developed satellite navigation systems are developing with a prime importance for compatibility and interoperability with the other already established satellite navigation systems. Receiver manufacturers also felt the importance of developing Multi-GNSS receivers. Cost- effective Multi-GNSS receivers are entering and capturing the market quickly. But, for multiple signal availability receivers used to operate in internal offset mode rather than using all the systems interchangeably which is rather difficult to achieve as virtual link between the systems is required beforehand. This manipulation technique by the receivers is unknown to user and lead to unreliable solution accuracy. This causes a highly receiver dependent result. A more precise globally accepted interoperability technique is required for having proper benefits of using Multi-GNSS.

\section{Acknowledgments}

I would like to acknowledge GNSS Laboratory, Department of Physics, The University of Burdwan for helping me in executing this research work.

\section{Conflicts of interest}

Authors declare that there is no conflict of interest.

\section{References}

1. Information and Analysis Centre for Positioning, Navigation and Timing. GLONASS News; 2005.

2. Constellation Information. European Global Navigation Satellite Systems Agency; 2020.

3. Hofmann-Wellenhof B, Lichtenegger H, Collins J. Global Positioning System: Theory and Practice. Springer-Verlag/Wien; 2001.

4. Teunissen PJG, Montenbruck O. Springer Handbook of Global Navigation Satellite Systems. Springer; 2017.

5. Hein G. GNSS Interoperability: Achieving a Global System of Sysetms or "Does Everything Have to Be the Same?" Inside GNSS; 2006. 57-60 p.

6. Galileo Ground Segment. European Space Agency; 2019.

7. Official U.S. government information about the Global Positioning System (GPS). GPS.GOV; 2018.

8. Bazlov AY, Galazin EV, Kaplan LB, et al. Propagating PZ 90 to WGS 84 Transformation Parameters. GPS Solutions. 1999;3:13-16.

9. Boucher C, Altamimi Z. PZ- 90 and WGS 84: Current realizations and the related transformation Parameters. Journal of Geodesy. 2001;75:613-619. 
10. Bykhanov E. Timing and Positioning with GLONASS and GPS. GPS Solutions. 1999;3:26-31.

11. Daly P, Mishra P. GPS and Global Navigation Satellite System (GLONASS). Global Positioning system: Theory and Applications. 1996;2:243-272.

12. Daly P, Koshelyaevsky BN, Lewandowski W, et al. 1993;30:89-94.

13. Report of Working Group A: Compatibility and Interoperability. ICG/ WGA; 2008.

14. Indian Regional Navigation Satellite System (IRNSS): NavIC. ISRO; 2017
15. China Satellite Navigation Office. BeiDou: Bring the World and China to your Doorstep. US, UNOOSA; 2012.

16. A Coordination methodology for RNSS intersystem inetrfernce estimation. International Telecommunication Union; 2015

17. Sarkar S, Mahato S, Santra A, et al. GLONASS: Present and Future. UN/ Fiji Workshop on Application of GNSS: Suva, Fiji; 2019.

18. Bose A. Studies on the Accuracy of Timing via Satellites and Positioning via GPS. Burdwan: India; 2002. 